\title{
GO++: A MODULAR LAGRANGIAN/EULERIAN SOFTWARE FOR HAMILTON JACOBI EQUATIONS OF GEOMETRIC OPTICS TYPE
}

\author{
JeAn-DaVid Benamou ${ }^{1}$ And Philippe $\mathrm{HOCH}^{1}$
}

\begin{abstract}
We describe both the classical Lagrangian and the Eulerian methods for first order HamiltonJacobi equations of geometric optic type. We then explain the basic structure of the software and how new solvers/models can be added to it. A selection of numerical examples are presented.
\end{abstract}

Mathematics Subject Classification. 78A05, 78H20.

Received: 5 December, 2001. Revised: 6 May, 2002.

\section{INTRODUCTION}

Ray tracing is a widespread numerical technique. Its is, for instance, routinely used in the oil industry to compute travel-times of seismic waves in the underground under the geometric optics approximation of wave propagation. Geometric optics is also a prototype problem for first order Hamilton-Jacobi equations when the Hamiltonian function enjoys convexity properties. This class of problems appears in rather diverse applications such as wave propagation, mesh generation, combustion, crystal growth and more generally in a large number of problems of the calculus of variations.

Ray tracing consists in solving a set of ordinary differential equations called the Hamiltonian systems. One of the variable describes a Lagrangian trajectory (the ray) along which the others, the Lagrangian variables, are transported. This approach is conceptually simple and numerically easy to use. However, as a Lagrangian method, it does not give any control on the spatial resolution of the Lagrangian solution. Indeed, the rays are necessarily in finite numbers and produce when diverging poor resolution. Various refinement procedures exist to fix this problem $[13,18]$ which are based on interpolation techniques.

In the Eulerian approach the main variable, defined on the configuration space, satisfies a Hamilton-Jacobi partial differential equation. It can be discretized on an a-priori fixed grid. The space resolution of the numerical method is therefore automatically maintained and the accuracy of the approximation depends on this resolution. These two approaches, Lagrangian and Eulerian, are mathematically equivalent only when the Lagrangian solution is well defined and single-valued (rays do not cross). The Eulerian method otherwise produces a weak "viscosity" solution which can be identified as the minimum value of the multi-valued Lagrangian solution (the problem of computing the Lagrangian solution using a Eulerian representation when it is multi-valued has been the subject of on-going work in the last ten years $[1,4,5,7,10,11,14,16,17] \ldots)$.

\footnotetext{
Keywords and phrases. Hamilton-Jacobi, Hamiltonian system, ray tracing, viscosity solution, upwind scheme, geometric optics, $\mathrm{C}++$.

${ }^{1}$ INRIA, Domaine de Voluceau, BP 105, 78153 Le Chesnay Cedex, France. e-mail: Jean-David.Benamou@inria.fr
} 
We review here the basic framework of the problem, introducing both Lagrangian and Eulerian solutions and their relations. Next we detail the structure of our software which is intended to provide a $\mathrm{C}++$ framework for an easy implementation, use and comparison of both Lagrangian and Eulerian numerical methods applied to Hamilton-Jacobi equations. We explain then how GO++ can be used to solve a standard Geometric Optics problem and modified to accommodate for a general Hamiltonian. Finally we give indications on how a new solver can be added to this modular software and again give an example. Even though large parts of GO++ are documented here, this paper is not a manual or a tutorial but rather a description of the choices we made to build the software.

We would like to point out that one of our purposes is to explore the use of $\mathrm{C}++$ for providing an efficient open source library for education and research. Scientific computing nowadays often combines several numerical techniques and makes software development rather difficult and time consuming. Instead of programming from scratch, meta-languages (such as Matlab ${ }^{\circledR}$ ) allowing a fast and easy (bug free) use of standard numerical algorithms are popular. However, theses libraries are too large to be fast enough for 2-D and 3-D "realistic" applications. A more efficient general scientific solver like FreeFem + (http://www-rocq.inria.fr/Frederic.Hecht/ FreeFemPlus.htm) uses a $\mathrm{C}++$ idiom as a wrapper. We want to simplify further the interface and we believe that it is possible to directly use the $\mathrm{C}++$ language. We think that a $\mathrm{C}++$ modular library which targets a particular class of application can be made "user-friendly" for researchers (used to programming but not necessarily $\mathrm{C}++$ experts) without any sort of "wrapping". The expected gain is the efficiency of the produced compiled code in term of running time.

\section{THE MODELS}

\subsection{The Lagrangian solution and the ray method}

A Hamiltonian function $H(s, y, p)$ is given, defined on $\mathbb{R}_{s}^{+} \times \mathbb{R}_{y}^{2} \times \mathbb{R}_{p}^{2}$ (we will generically set $y=\left(y_{1}, y_{2}\right)$, $\left.p=\left(p_{1}, p_{2}\right) \ldots\right) ; \mathbb{R}_{s}^{+} \times \mathbb{R}_{y}^{2}$ is the time-space configuration in which rays can evolve and $\mathbb{R}_{s}^{+} \times \mathbb{R}_{y}^{2} \times \mathbb{R}_{p}^{2}$ is called the phase space.

The Lagrangian method consists in solving (using an ODE solver) the Hamiltonian system formed by the following set of ordinary differential equations (ODEs) $[2,9,12,19]$ :

$$
\left\{\begin{array}{l}
\dot{y}\left(s, y^{0}\right)=H_{p}\left(s, y\left(s, y^{0}\right), p\left(s, y^{0}\right)\right), y\left(0, y^{0}\right)=y^{0} \\
\dot{p}\left(s, y^{0}\right)=-H_{y}\left(s, y\left(s, y^{0}\right), p\left(s, y^{0}\right)\right), p\left(0, y^{0}\right)=\phi_{y^{0}}^{0}\left(y^{0}\right) \\
\dot{\varphi}\left(s, y^{0}\right)=p\left(s, y^{0}\right) \cdot H_{p}\left(s, y\left(s, y^{0}\right), p\left(s, y^{0}\right)\right)-H\left(s, y\left(s, y^{0}\right), p\left(s, y^{0}\right)\right) \\
\varphi\left(0, y^{0}\right)=\phi^{0}\left(y^{0}\right)
\end{array}\right.
$$

The dot stands for derivation with respect to "time" $()=.\frac{\mathrm{d}(.)}{\mathrm{d} s}, g_{x}\left(x_{1}, x_{2}\right)$ denotes the gradient with respect to $x=\left(x_{1}, x_{2}\right), \phi^{0}$ is a given initial phase and also appears in the initial condition for $p$. For each $y^{0} \in \mathbb{R}_{y}^{2}$ (or a subset of $\mathbb{R}_{y}^{2}$ ), the system generates a "bicharacteristic strip" $\left(y\left(s, y^{0}\right), p\left(s, y^{0}\right)\right)$ depending on $s$ and $y^{0}$. The projections of the strips onto $\mathbb{R}_{y}^{2}: y\left(s, y^{0}\right)$, are called the rays. Each ray is therefore "labeled" by its initial position $y^{0}$. The phase $\varphi\left(s, y^{0}\right)$ is transported by the corresponding ray $y\left(s, y^{0}\right)$ and, when rays are crossing, is a multi-valued function of the configuration space $\mathbb{R}_{s}^{+} \times \mathbb{R}_{y}^{2}$.

\subsection{The Eulerian solution and the Hamilton-Jacobi partial differential equation}

As we proceed along the ray and as long as rays do not cross, we can apply a local inversion theorem to the mapping $y^{0} \rightarrow y\left(s, y^{0}\right)$. Assuming that every point $(s, x) \in \mathbb{R}_{s}^{+} \times \mathbb{R}_{y}^{2}$ is reached by only one ray, we can introduce the Eulerian variable $\phi(s, x)$ which, evaluated at the Lagrangian coordinates specified by the rays, 
matches the Lagrangian phase:

$$
\phi\left(s, y\left(s, y^{0}\right)\right)=\varphi\left(s, y^{0}\right)
$$

It is possible to derive a Hamilton-Jacobi equation satisfied by $\phi(s, x)$ either in the classical sense when rays do not cross or in the viscosity sense $[3,8]$ else

$$
\left\{\begin{array}{l}
\frac{\partial \phi}{\partial s}(s, x)+H(s, x, \nabla \phi(s, x))=0, \text { for }(s, x) \in \mathbb{R}_{s}^{+} \times \mathbb{R}_{y}^{d} \\
\phi\left(0, y^{0}\right)=\phi^{0}\left(y^{0}\right), \text { for } y^{0} \in \mathbb{R}_{y}^{d} .
\end{array}\right.
$$

A link can be made between Lagrangian and Eulerian viscosity solution using the theory of optimal control [5]. The viscosity solution can be characterized as the minimum of the associated Lagrangian phases:

$$
\phi(s, x)=\min _{y^{0}, \text { s.t. } y\left(s, y^{0}\right)=x} \varphi\left(s, y^{0}\right) .
$$

If there is a zone where no ray penetrates, the viscosity solution implicitly generates "non-classical" rays to fill this empty zone. It means that the optimal curves will satisfy the Hamiltonian system (1) with initial conditions different from those specified for classical rays. This situation seems to be linked to diffraction phenomena and should hopefully be investigated elsewhere.

\subsection{Domain and boundary conditions}

In practice, computations are limited to a given bounded domain and we must enforce boundary conditions. The simplest condition for rays is to ignore them as soon as they exit the domain. It corresponds to an "out-going" boundary condition for the Hamilton-Jacobi equation. It is implemented by the enforcement of the continuity of the flux across the boundary (for instance for Lax-Friedrich solvers) or more simply, in the case of Godunov solver by the Dirichlet boundary condition $\phi=+\infty$ (in practice $\phi=M, M$ large enough) (Soner condition). More generally, we want to be able to treat general Dirichlet, Neumann or mixed boundary conditions which can be for instance interpreted in the Lagrangian case as reflecting laws for the rays (see [3] for more on boundary conditions).

\subsection{Initialization}

The simplest Eulerian initialization (the default initialization) is $\phi^{0}\left(y^{0}\right)=0$ in $(3)$. In term of rays, it means that they start with a direction $p=0$ from all points of the domain.

An other common initialization for ray tracing is the isotropic point source. All rays start at a source point $S$ with direction $p$ on the unit circle. We can use a Eulerian initialization using the distance function from $S$ : $\phi^{0}\left(y^{0}\right)=\left\|S-y^{0}\right\|$ to obtain a Eulerian emulation of the Lagrangian isotropic source initialization.

\section{The MAIN StRuCture OF $\mathrm{GO}++$}

The computational kernel of $\mathrm{GO}++$ is more general that is needed for our purpose. It is designed to solve a set of ordinary differential equations

$$
\dot{X}(s)=R H S(X(s))
$$

where $X(s)$ is a collection of variables and $R H S$ a function depending on the Hamiltonian, the boundary conditions and the discretization. We recall that $\mathrm{GO}++$ must allow Lagrangian and/or Eulerian computations. We therefore decided to define a unique data structure in a class called ISH for solving (5). A member of this class $X$ represents either a set of bicharacteristics and associated phases: $(X . y, X . p, X . p h i)=(y, p, \varphi)$ at a fixed time $s$ and (5) represents the Hamiltonian system (1) or, in the Eulerian framework, $X . y=x$ stands 
for the discretization points while $(X . p, X . p h i)=(\nabla \phi(s, x), \phi(s, x))$ are respectively the gradient of the phase and the phase itself at those points. In this case the equation satisfied by $X$.phi in (5) corresponds to the Hamilton-Jacobi equation (3).

This approach is obviously not optimal in the Eulerian case alone because the remaining equations in (5) are not a priori needed and the data could resume to a simple scalar function $\phi$. In our opinion these drawbacks are compensated by the following advantages:

- This setting allows to define a unique class describing the Hamiltonian in which a function called $H x p h$ maps the ISH data $X$ described above to another ISH data $Y$ which stands for $\left(H_{y}(s, y, p), H_{p}(s, y, p)\right.$, $H(s, y, p))$. At a fixed time $s$, the same data structure can be used for $X$ and $Y$.

- The Hamiltonian function may be straightforwardly generalized to depend also on $\phi: H(s, y, p, \phi)$.

- The time solver modules used to solve (5) can be applied in Lagrangian and Eulerian modes.

- In the Eulerian mode, $y=x$ and $p=\nabla \phi(s, x)$; the additional quantities $\left(H_{y}(s, x, \nabla \phi(s, x)), H_{p}(s, x\right.$, $\nabla \phi(s, x))$ are needed to implement some Hamilton-Jacobi solvers.

- The data $X . y$ representing the grid points and the associated equation in (5) could be used to implement a moving or adaptative grid.

The software consists of independent modules in the the form of $\mathrm{C}++$ classes. Those of these classes which prescribe the problem or the numerical methods are virtual classes containing virtual functions. Adding new problems or new numerical methods to $\mathrm{GO}++$ consists in writing or modifying existing children classes of the virtual classes. We now describe the basic classes needed to get started.

\section{Domain, Discretization And DATA StruCture}

The software is for now restricted to a rectangular 2-D spatial domain and regular Cartesian discretizations. This choice simplifies the implementation of finite difference solvers.

In order to generalize the software to treat 3-D problems or unstructured grid, the class presented in this section must be modified. Even though it may be possible to treat such generalization by creating virtual geometry, discretization and data classes, we believe that a better (and simpler) solution is to create several versions of the software, each one dedicated to a particular discretization and to 2D or 3D problems.

\subsection{Domain: the $G E O M$ class}

The GEOM class describes the geometry of the problem. It is used to define rectangular domains.

The declaration GEOM geometry $\left(x_{1, \min }, x_{1, \max }, x_{2, \min }, x_{2, \max }\right)$ creates a regular 2-D rectangular geometry by means of the $x_{1}$ and $x_{2}$ coordinates of its corners: $x_{1, \min }, x_{1, \max }, x_{2, \min }, x_{2, \max }$.

\subsection{Discretization: the $D S C R$ class}

The $D S C R$ class describes the discretization of the problem. We will consider that in the Eulerian framework the discretization stands for the set of grid points at which the solution will be computed. In the Lagrangian mode, the discretization will be the set of initial points for the rays. The DSCR needs a GEOM class to build this set of points. The accuracy of the Eulerian solution depends on the number of discretization points and also on the expected "order" of accuracy of the numerical methods to be used. An integer ord specify the order and sets the number of points of the external layer used for computing finite differences at the boundary.

The declaration DSCR discretization (geometry, $n x_{1}, n x_{2}$, ord) can be used both in Lagrangian and Eulerian mode. It specifies the number of discretization points $\left(n x_{1}, n x_{2}\right)$ in each coordinate direction. It builds the regular 2-D Cartesian grid of points $\left(x_{1, i}, x_{2, j}\right), i=1, . ., n x_{1}+2 *$ ord $, j=1, . ., n x_{2}+2 *$ ord needed to implement a finite difference method of order ord with $n x_{1} \times n x_{2}$ points covering the domain specified by the GEOM class or one of its children (note that the actual domain is spanned by the set of points $\left(x_{1, i}, x_{2, j}\right), i=$ ord,..,$n x_{1}+$ ord $-1, j=$ ord,..,$n x_{2}+$ ord -1$)$. 
In the Lagrangian mode, it determines the size of the arrays which will describes number of rays we use $\left(n x_{1} \times n x_{2}\right)$. It can also be used for a default initialization of the initial points $y^{0}=\left(x_{1, i}, x_{2, j}\right), i=1, . ., n x_{1}, j=$ $1, \ldots, n x_{2}$. The general initialization process is described in Section 5 .

\subsection{Data structure: the $\boldsymbol{I S H}$ class}

The discretization class provides the necessary information for defining the data structure of a member $X$ of $I S H$. It will typically be three arrays which numbers of dimensions and sizes are determined by the discretization of the problem.

The basic data structure is made of two $2 \times n x_{1} \times n x_{2}$ arrays of reals called X.y and X.p and one $n x_{1} \times n x_{2}$ array of reals called X.phi.

$I S H$ is built on top of a collection of freely available array classes: $R N M$ (see http://www-rocq.inria.fr/ Frederic.Hecht). These classes are much simpler than other packages (such as Blitz), easy to compile and the functionalities (vectorial Matlab-like operations, scalar products, ...) sufficient for our purpose.

ISH also contains utility member functions for reading and writing at any time step $s$ either the collection of $\left(y^{0}, \varphi_{x}^{0}\left(s, y^{0}\right), \varphi^{0}\left(y^{0}\right)\right)$ for the rays or of the Eulerian variables $(x, \nabla \phi(s, x), \phi(s, x))$. It uses the overload of "«" (used for the output) in the RNM class.

When an $I S H$ variable is declared, the default initialization is $X=0$. There is however the possibility of specifying a function $\phi^{0}$ in the initialization list. The function maps an array $X$ describing either $y^{0}$ or $x$ into an ISH Y for which you have to specify (Y.y $=\mathrm{X}$ of course) $Y . p h i=f(X)$ and Y.p $=f_{x}(X)$ (see next section).

\section{Describing functions of the configuration $(x)$ space: the $X F C T$ Class}

We first need a virtual class XFCT which describes a function of the configuration space. This class has a virtual function called $F$ which takes an ISH variable FX for which FX.y is a $2 \times n_{x_{1}} \times n_{x_{2}}$ array describing a set of points in the domain. The remaining variables $F X . p=\nabla F(X)$ is the gradient of the function at these points and $F X . p h i=F(X)$ the function itself.

The class has two families of children in which the $X F C T$ can be specialized. In the $X F C T \_A N A L$ class the function is specified analytically. In the second class $X F C T \_I N T R$, the function is specified by its evaluation at given configuration points. To build such an object, we must read data in the form of two arrays (the specification of sample points and the value of the function at these points) and we need an interpolation method to be able to evaluate the XFCT on any array of points (more details on this class in Sect. 11.1.)

As an example, suppose we want to initialize a Lagrangian problem where all the rays start at a source point $S$, with initial direction on the sphere. We need to define an XFCT child class called XFCT_INIT_LAG_SRCP for which $S$ is given as a data member.

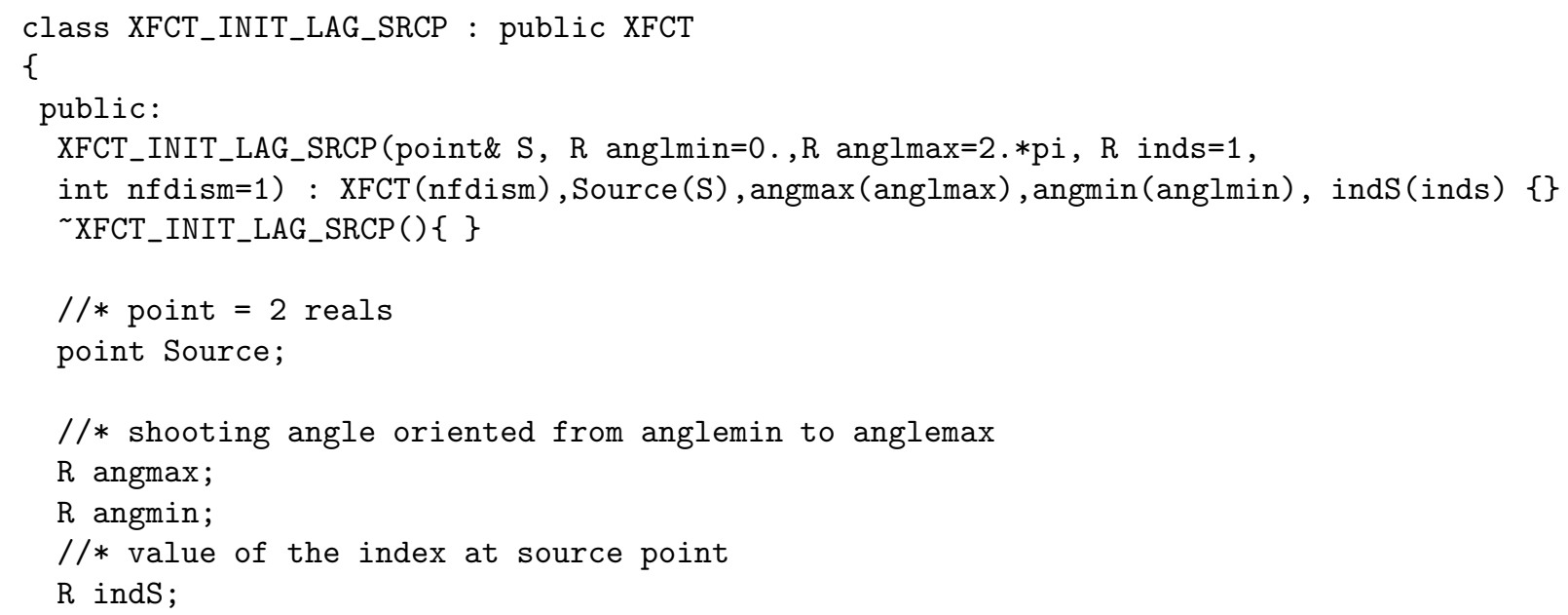


\} ;

void F(ISH* FX) const;

Then the $F$ function, is specialized in the following $\mathrm{C}++$ source:

//* specializes the $\mathrm{F}$ function of the new class

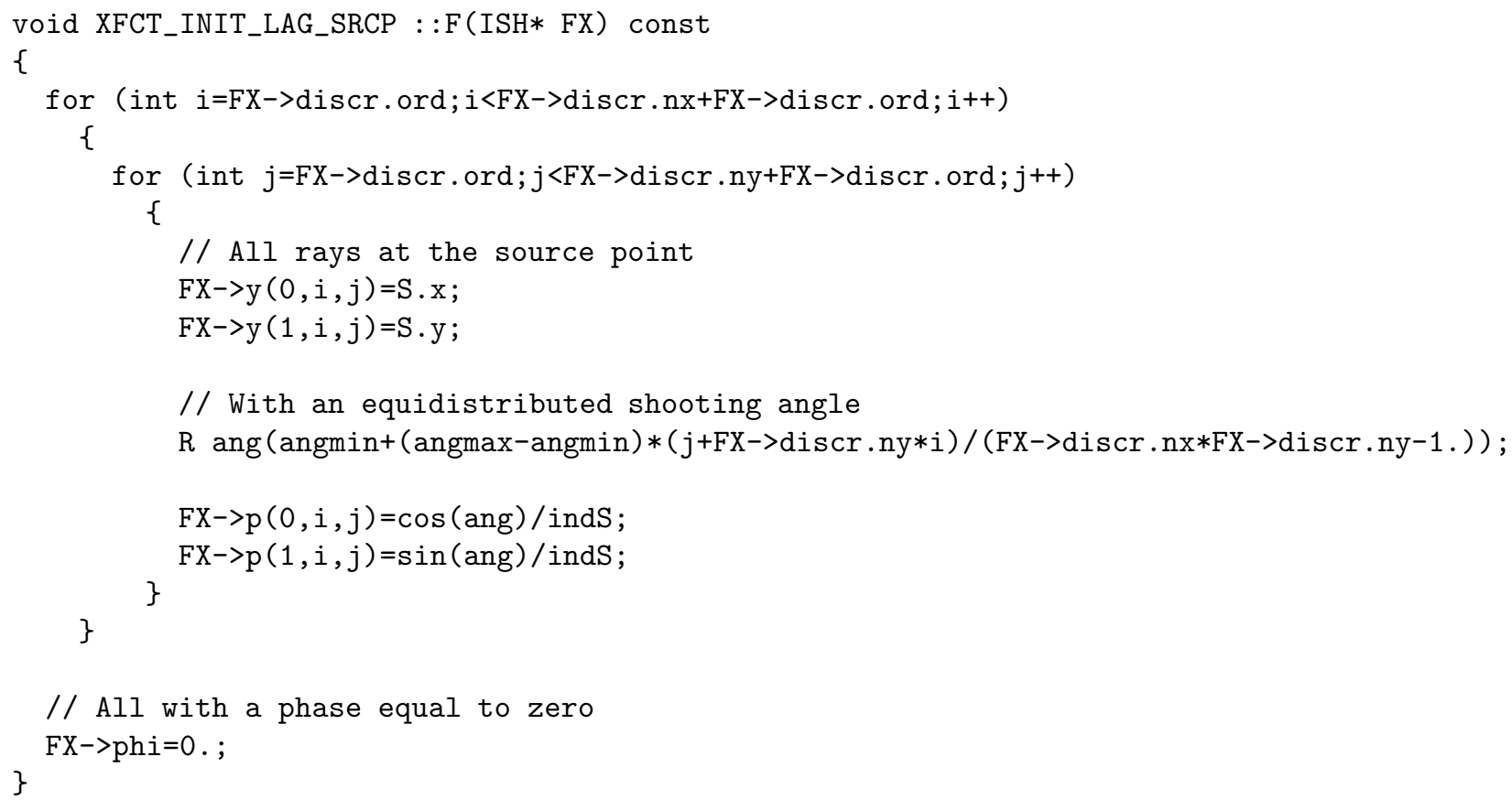

As discussed in Section 2.4, the corresponding behavior in the Eulerian mode can be emulated by the initial function $\phi^{0}(X)=\|S-X\|$. The implementation of this function is similar. We create the child $X F C T \_I N I T \_E U L \_S R C P$ for which $S$ is given as a data member.

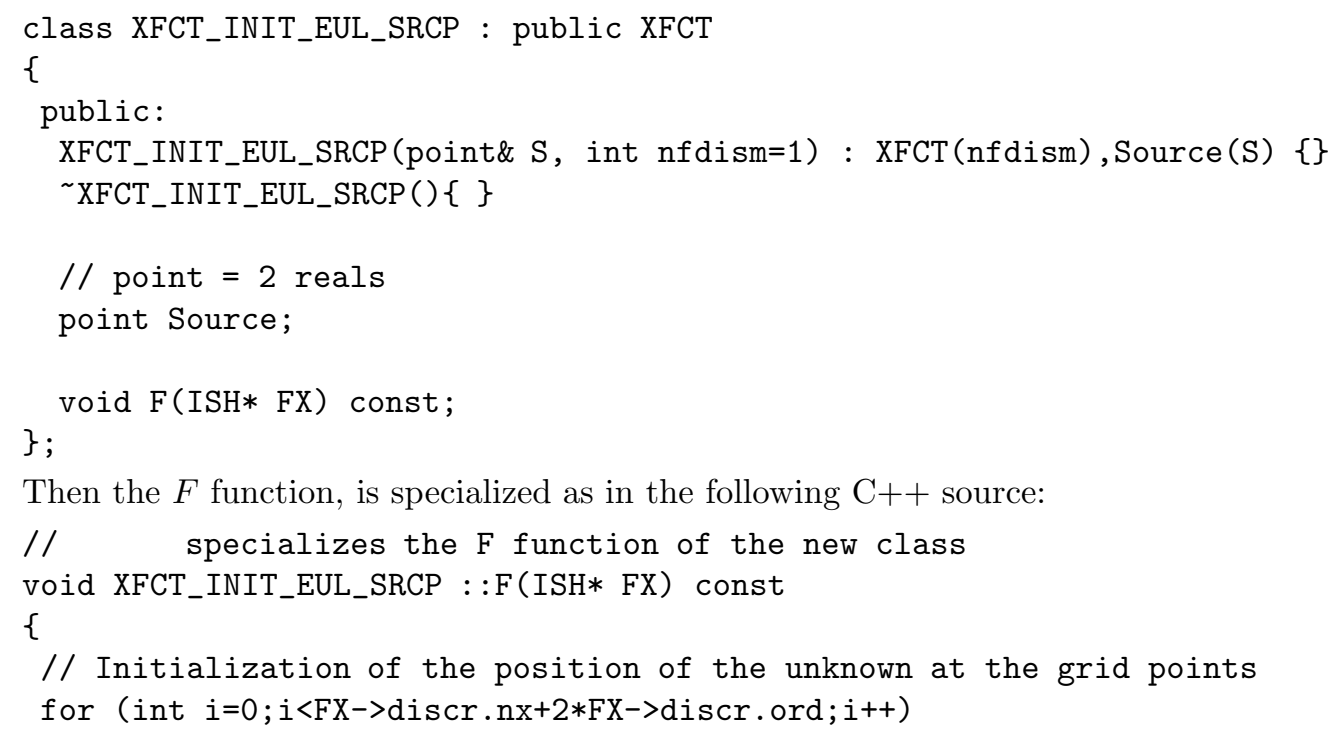




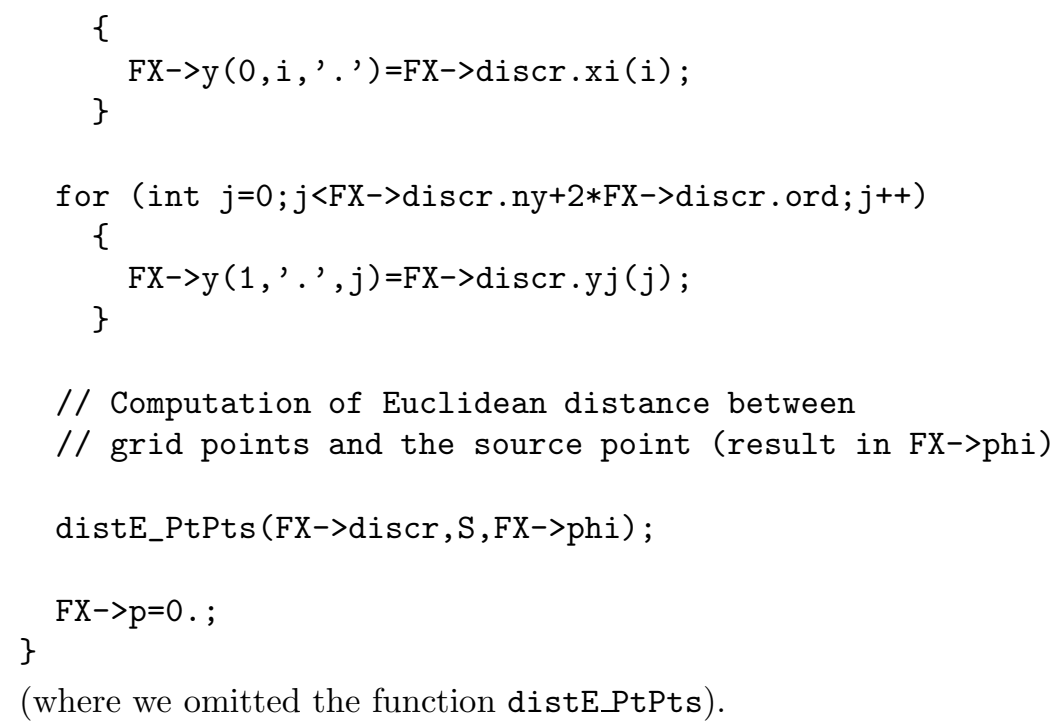

(where we omitted the function distE_PtPts).

\section{THE MODEL}

\subsection{Boundary conditions: the $B C O N$ class}

The boundary condition class can be used either to implement classical boundary conditions in the Eulerian case or to specify the behavior of rays when the limit of the computational domain is reached. The virtual class $B C O N$ has a virtual member function $S b c o$ which uses information from the $D S C R$ class (discretization) to modify an $I S H$ variable. In the Lagrangian mode, Sbco modifies the $I S H$ standing for the $R H S$ in (5) for those of the rays which exit the domain. When solving a Eulerian problem, boundary conditions are implemented by filling the boundary layer (one or more points) with correct values of the ISH representing the current solution. The width of the boundary layer is set by the order ord.

The simplest boundary conditions are "out-going" boundary conditions as described in Section 2.3. It is implemented in the child classes $B C O N_{-} L A G \_O U T G, B C O N \_E U L_{-} O U T G \_L A X F R$ for Lax-Friedrich solvers and BCON_EUL_OUTG_GODUNOV for Godunov solvers.

// Here, X represents the solution, and $Y$ the flux.

void BCON_LAG_OUTG: :Sbco(const R\& $t$, ISH* X,ISH\& Y) const

\{

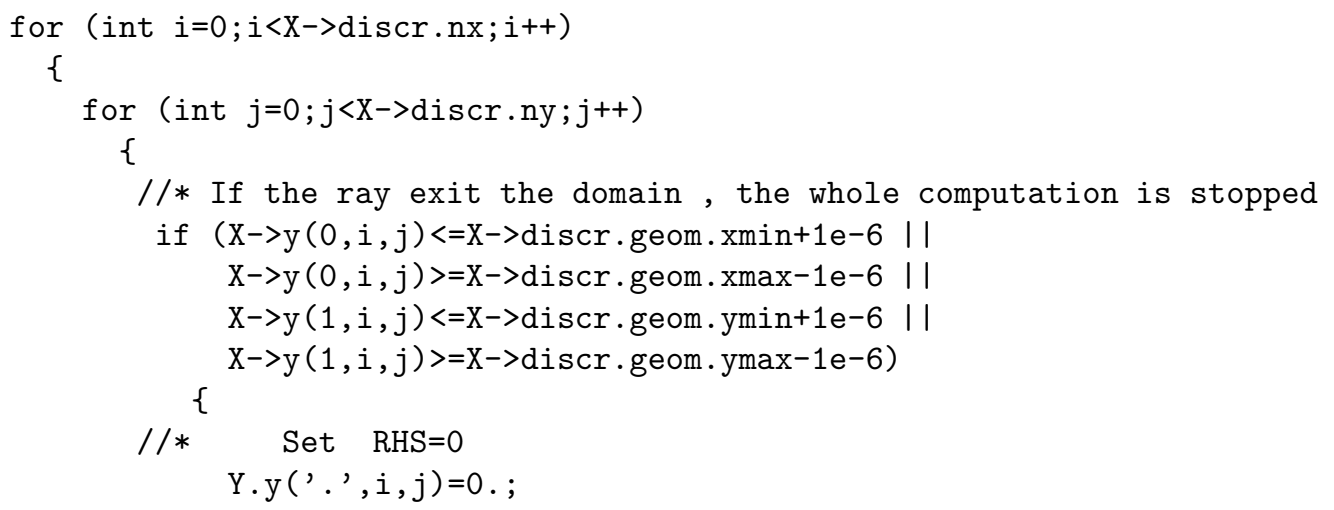




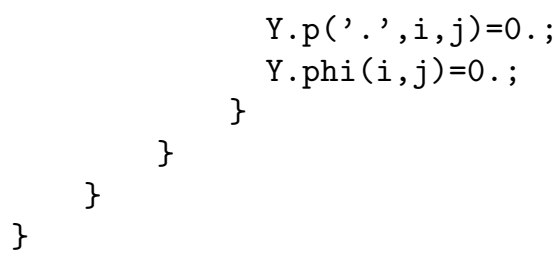

and in the Eulerian mode (for Godunov type solvers)

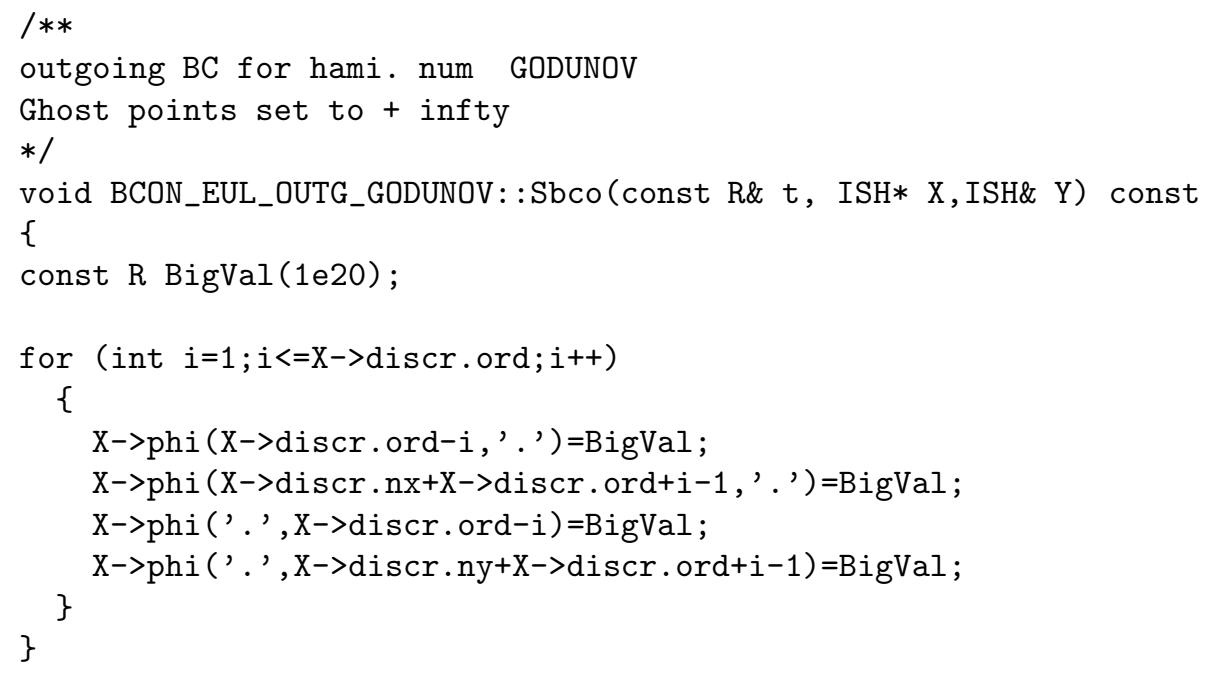

\subsection{Hamiltonian function: the $H A M I$ class}

The Hamiltonian function $H(t, y, p)$ is the central object of the problem and is represented through a virtual class $H A M I$. As discussed in Section 3, a virtual function $H x p h$ maps an $I S H$ data $X$ to another $I S H$ data $Y$. The $H A M I$ hierarchy of class has two main branches.

In the first branch called $H A M I_{-} C O N T$, the $(X . y, X . p, X . p h i)$ variables are the set of bicharacteristics and phase at a fixed "time" $s$ and the $Y$ data stands for the corresponding set of "continuous" evaluations of $\left(H_{y}(s, X . y, X . p), H_{p}(s, X . y, X . p), H(x, X . y, X . p)\right)$ needed to solve the Hamiltonian system (1). It can be straightforwardly used for Lagrangian computations.

In the Eulerian case, the space discretization of the Hamilton-Jacobi equation needs a second branch called $H A M I_{-} N U M$ described in Section 7.1.

One of the simplest H-J function is analytically specified by $H(s, y, p)=0.5 *\|p\|^{2}$. It is contained in the child class $H A M I_{-} C O N T \_A N A L \_P 2$. It is implemented in the following piece of $\mathrm{C}++$ header

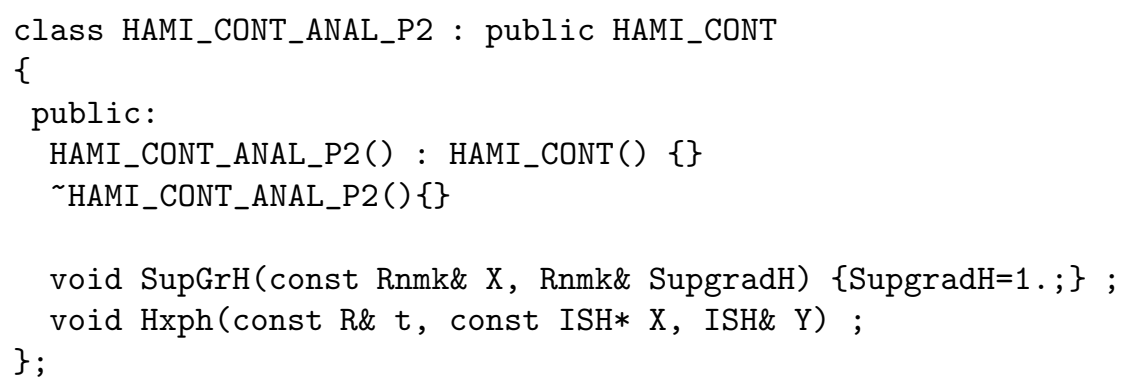


and the code

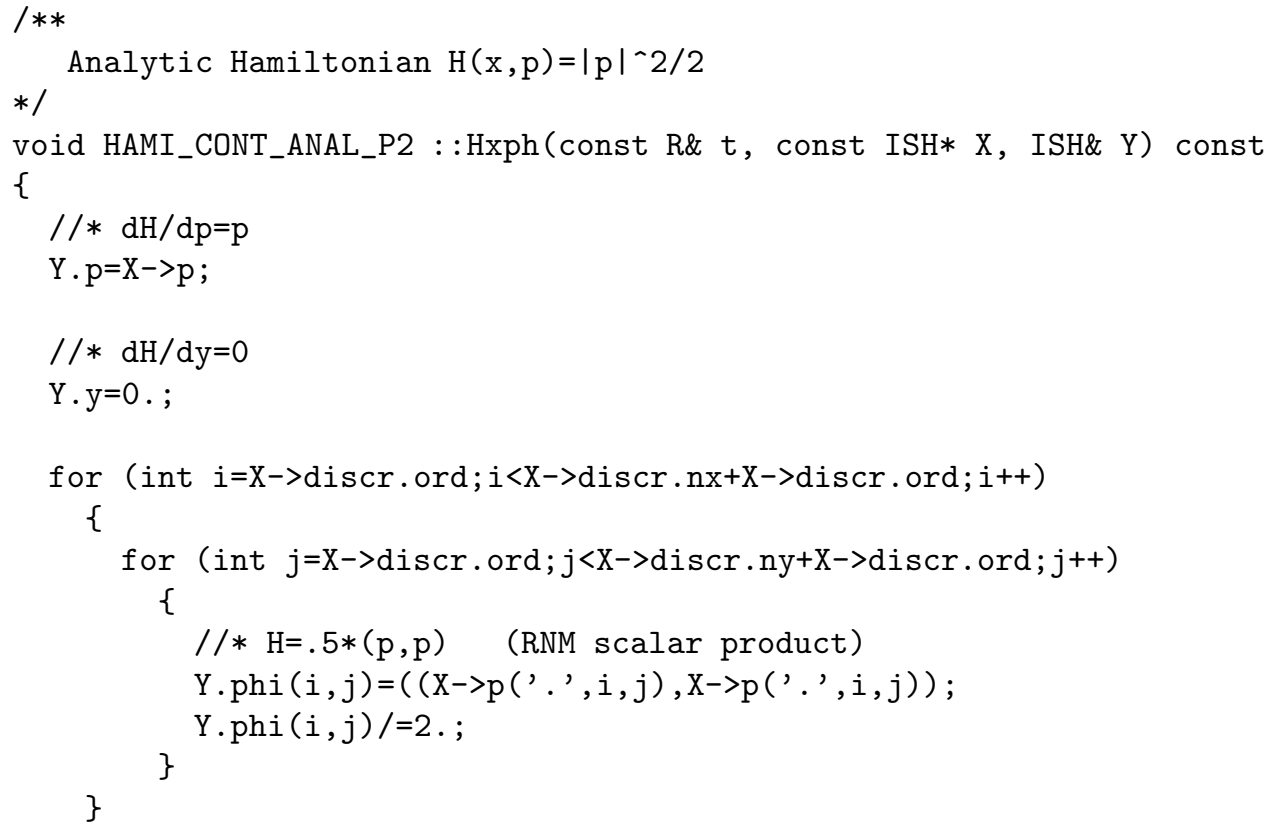

\section{Numerical methods}

\subsection{Space solvers, the $H A M I_{-} N U M$ and $G R A P$ classes}

This section only concerns the Eulerian approach even though one could think of further development in the Lagrangian case which would require an enrichment of the $H A M I$ structure (symplectic solvers for instance).

In the Eulerian framework, the ISH data $(X . y, X . p)$ stands for $(x, \nabla \phi(s, x))$ and we need to specify a method for approximating the gradient of the phase, i.e. to numerically implement the affectation $X . p=\nabla \phi(s, x)$. This is the object of the GRAP class. As "upwinding" is a classical tool in this context a member function Grphi takes the $n x_{1} \times n x_{2}$ array representing the phase $X$.phi as an input and returns two $2 \times n x_{1} \times n x_{2}$ arrays Grphim $=(a, c)$ and Grphip $=(b, d)$ which are the discrete upwind derivatives of order ord: if ord $=1$ and $\phi\left(s, x_{1, i}, x_{2, j}\right) \simeq \phi_{i, j}$ then $a=\frac{\left(\phi_{i, j}-\phi_{i-1, j}\right)}{\Delta x_{1}}, b=\frac{\left(\phi_{i+1, j}-\phi_{i, j}\right)}{\Delta x_{1}}, c=\frac{\left(\phi_{i, j}-\phi_{i, j-1}\right)}{\Delta x_{2}}, d=\frac{\left(\phi_{i, j+1}-\phi_{i, j}\right)}{\Delta x_{2}}$.

The simplest child class of GRAP is GRAP_UPW1 which computes upwind finite difference of order 1 (the $(a, b, c, d)$ quantities above). Other types of approximation such a TVD or ENO are available.

The discretization of (3) may also require some modifications of the Hamiltonian function itself. We therefore created a virtual child class of $H A M I$ called $H A M I_{-} N U M$ which uses the specifications of a given $H A M I \_C O N T$ Hamiltonian function and a method of approximation of $\nabla \phi$ (a GRAP class) to evaluate its own "numerical" Hxph function.

A simple method is the Lax-Friedrichs solver which replaces $H(s, x, \nabla \phi(s, x))$ by

$$
H_{L F}^{n u m}(s, x, a, b, c, d):=H\left(s, x, \frac{a+b}{2}, \frac{c+d}{2}\right)-\frac{\alpha(x)}{2}(b-a)-\frac{\beta(x)}{2}(d-c),
$$

where $(a, c)$ resp. $(b, d)$ are defined above, is designed by the $H A M I_{-} N U M_{-} L A X F$ class. The coefficients $\alpha(x):=\sup _{(u, v)}\left|\frac{\partial H}{\partial u}\right|$, and $\beta(x):=\sup _{(u, v)}\left|\frac{\partial H}{\partial v}\right|$ are the maximum of the speeds $\left((u, v)\right.$ stands for the $\left(\phi_{x_{1}}, \phi_{x_{2}}\right)$ variables). They are computed in a function called $S u p G r H$ which is a virtual member of the $H A M I \_N U M$ class. 
The $H A M I_{-} N U M$ and $H A M I_{-} N U M_{-} L A X F$ class of $H A M I$ look like:

class HAMI_NUM : public HAMI

\{

public:

HAMI_NUM(HAMI_CONT \&H, const GRAP\& ApGrad) : HAMI (H.nf), Hamc(H), ApxGrad(ApGrad) \{

Grphip=new Rnmk (2,ApGrad.discr.nx+2*ApGrad.discr.ord,ApGrad.discr.ny+2*ApGrad.dis cr.ord);

Grphim=new Rnmk (2,ApGrad.discr.nx+2*ApGrad.discr.ord,ApGrad.discr.ny+2*ApGrad.dis cr.ord);

\}

Xmod=new ISH (ApGrad.discr);

virtual HAMI_NUM() \{delete Grphip;delete Grphim;delete Xmod;\}

//* The continuous Hamiltonian that we have to discretized

HAMI_CONT \&Hamc ;

//* The type of approximation of the gradients (TVD, ENO ...)

const GRAP \&ApxGrad ;

//* current upwind derivatives

Rnmk* Grphip;

Rnmk* Grphim;

//* pointer on modified solution (only the gradient is different)

ISH* Xmod;

//* ACCESS FUNCTION TO FX

//* -----------------------

//* The access function to FX is overloaded,

$/ / *$ it permits to use the SAME FX variable in the

//* Numerical Hamiltonian and the continuous one,

//* but only the latter has got a real meaning.

ISH $* \&$ fX() \{return Hamc.fX();\}

//* MODIFICATION FUNCTION TO FX

//* - ----------------

$/ / *$ In the case of a Numerical Hamiltonian

//* we don't re compute the $\mathrm{X}$ fields (the grid is fixed).

//* here FX is set to GridVal (fixed grid and values on it) at

$/ / *$ the level of rhs in the application, then here, it doesn't

$/ / *$ do anything.

inline void $x$ FHami (ISH* FX) \{\}

//* The SupGrH is overloaded like the access function $f X()$ to 
void SupGrH(const Rnmk\& X, Rnmk\& SupgradH) \{Hamc.SupGrH(X,SupgradH); ;

\}

then we can specified the Lax-Friedrichs as a child class of $H A M I \_N U M$

class HAMI_NUM_LFX : public HAMI_NUM

\{

public:

HAMI_NUM_LFX (HAMI_CONT \&H, const GRAP\& ApGrad) : HAMI_NUM(H,ApGrad) \{

// Compute and stores maximum speeds for the Hamiltonian

Hamc. $f X()=$ new ISH [Hamc.nf] (ApGrad.discr, 0.) ;

Hamc.SupGRADH ()=new Rnmk (2,ApGrad.discr.nx+2*ApGrad.discr.ord, ApGrad.discr.ny+2*ApGrad.discr.ord);

Hamc . SupGrH (Hamc . fX() [0] . y, *Hamc . SupGRADH ( )) ;

\}

delete[] Hamc.fX();

〜HAMI_NUM_LFX () \{delete Hamc.SupGRADH();\} \};

void Hxph (const R\& $t$, const ISH* X, ISH\& Y) ;

and the code for the numerical Hamiltonian

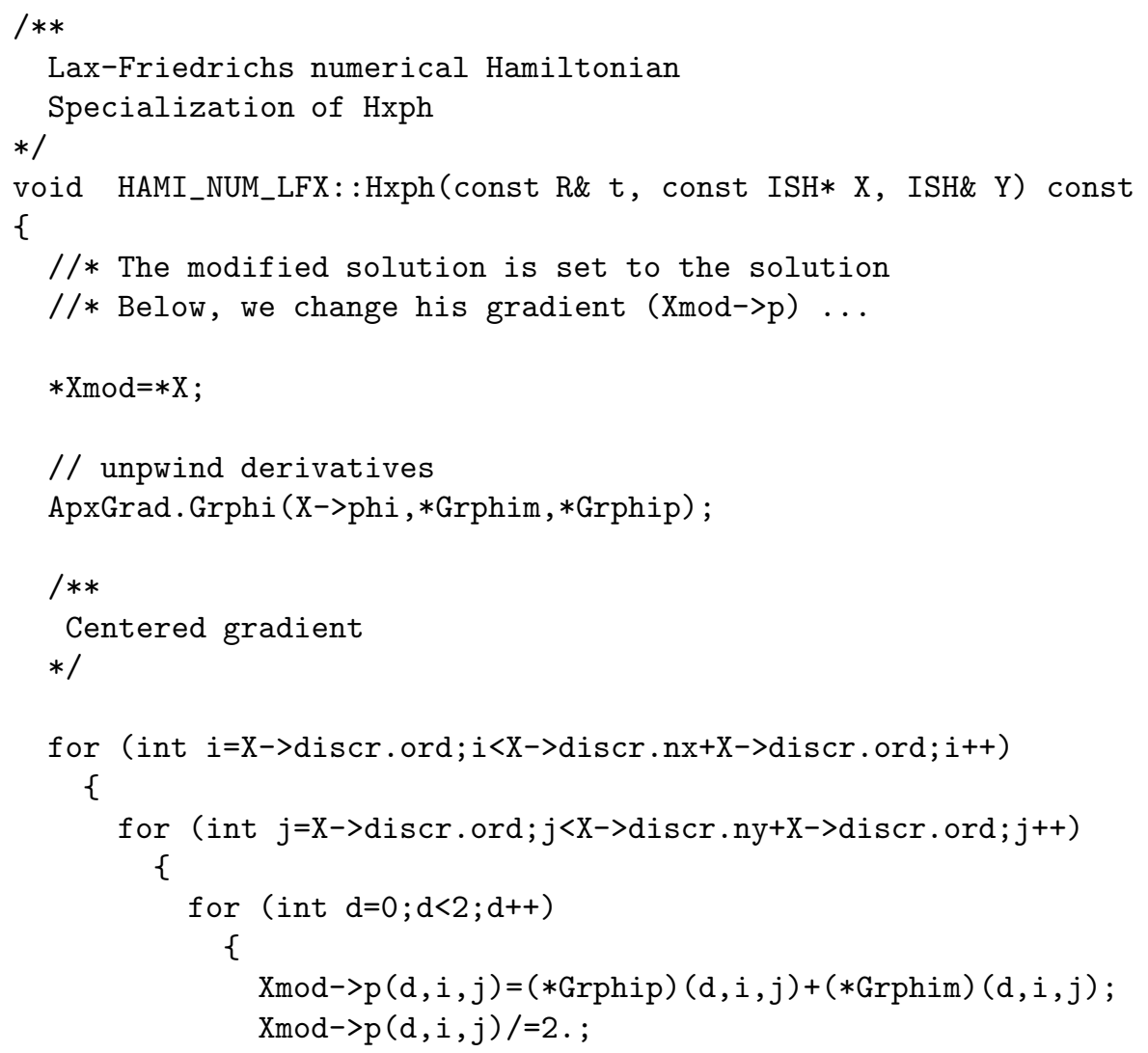




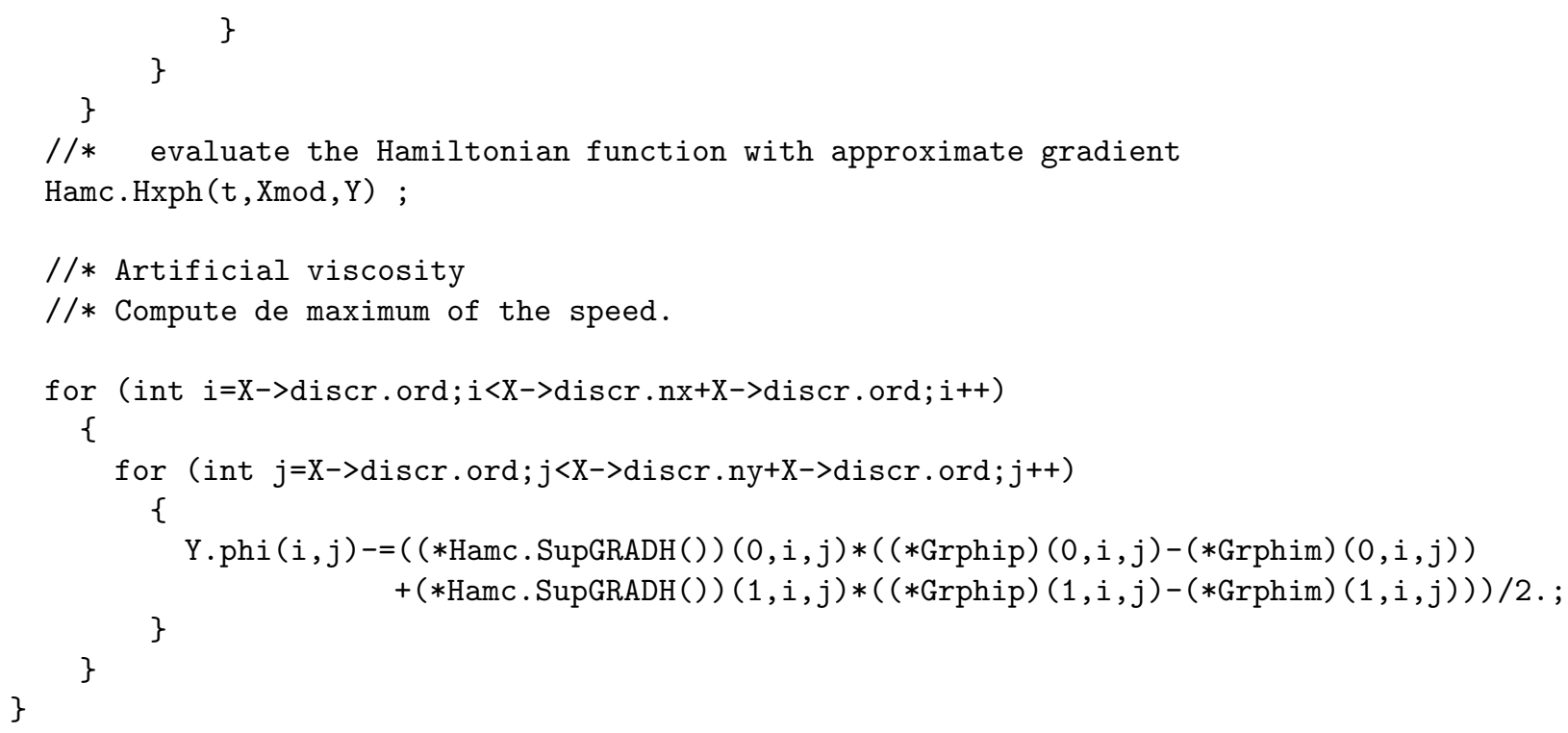

\subsection{Time solver, the $T S L V$ class}

Time solvers are designed to solve the set of ODE's (5) satisfied by an ISH unknown that has to be declared and initialized (see the $I S H$ section). It also assumes that a particular Rhs function is identified through the declaration of the type of application (see the APLI section below). The TSLV virtual class has a virtual member function Tstep which solves (5) on a time step $\mathrm{d} t$ (e.g. it takes the $I S H$ unknown $X(t)$ and the scalar $\mathrm{d} t$ and return $X(t+\mathrm{d} t)$. We already mentioned the structure of $\mathrm{GO}++$ makes it possible to use time solver for Lagrangian or Eulerian problems. The discretization is passed to time solver first for optimization purposes. It also provides the possibility to modify the discretization in time.

The simplest time solver is the Euler method $X(t+\mathrm{d} t)=X(t)+\mathrm{d} t * R H S(X(t))$ and is implemented in the child class $T L S V \_E U L$. Several other methods of Runge-Kutta type are also available.

\section{Defining the type of application: the APLI Class}

Once a discretization and a model is fixed, an ISH variable $X$ can be declared which represents the solution of the problem at a fixed time. It is then necessary to specify the type of application (e.g. Lagrangian or Eulerian) we wish to solve. It is done through the specialization of the $R h s$ function on the right hand side of (5).

Thus there exist a virtual class $A P L I$ of which $R h s$ is a virtual member function. It can be specialized into (at least) two child classes $A P L I_{\_} L A G$ and $A P L I_{\_} E U L_{-} E V$ for Lagrangian and Eulerian evolution applications. The function $R h s$ of course needs information on the model characterized by an Hamiltonian function $H x p h$ and boundary conditions $B C O N$. In the Lagrangian mode the boundary conditions are enforced (calling the Sbco function) after the evaluation of Rhs while in the Eulerian mode it is before (see Sect. 5.1).

\section{The MAIN PROGRAM: A FIRST EXAMPLE}

This section shows how all these objects can be combined to produce a simple Lagrangian/Eulerian computation for the Hamiltonian $H(s, y, p)=0.5 *\|p\|^{2}$ on a rectangular domain with outgoing boundary conditions, artesian discretization and point source initialization. This following source code is a prototype main program for $\mathrm{GO}++$ applications. Changes in domain, discretization, initialization, Hamiltonian functions, numerical methods, ..., can be implemented separately in child classes of the basic classes. The following sections detail such changes. 
\#include <iostream>

\#include <fstream>

\#include <assert.h>

\#include <math.h>

\#include <utility>

\#include <algorithm>

\#include <GO++.hpp $>$

/*

A typical main program for

Eulerian and Lagrangian computations $* /$

int main(int argc, char **argv)

\{

/******** Domain and discretization define a $2 \mathrm{D}$ rectangular domain with $(\mathrm{x}, \mathrm{y})$ coordinates of the corners

*/

$\mathrm{R} \operatorname{xmin}(0),. \operatorname{xmax}(4),. y \min (0),. y \max (2$.$) ;$

GEOM geom (xmin, xmax, ymin, ymax);

// Define a discretization of the domain

// Eulerian case

int $\mathrm{nxE}$ (51), $\mathrm{nyE}(51)$, ord (1);

DSCR discrE (geom, nxE, nyE, ord);

// Lagrangian case

int $\mathrm{nxL}(5), \mathrm{nyL}(5)$;

DSCR discrL (geom, nxL, nyL);

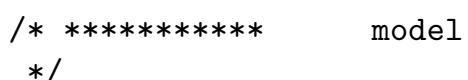

$* /$

// Boundary Conditions: Eulerian Case BCON_EUL_OUTG_LAXFR bcond;

// Boundary Conditions: Lagrangian Case BCON_LAG_OUTG bclag;

// Hamiltonian

HAMI_CONT_ANAL_P2 ham;

/***** Numerical methods in space for HJ eq.

// Type of approximation of grad phi

GRAP_UPW1 gradap(discrE);

// Numerical Hamiltonian (space) Lax-Friedrich 
HAMI_NUM_LFX hamnum(ham,gradap);

// ***** type of application $* * * * *$

// define type of RHS

// Eulerian Evolution application

APLI_EUL_EV eul (hamnum, bcond);

// Lagrangian application

APLI_LAG lag(ham,bclag);

// ****** define and initialize by XFCT

// Source Point

point $S$;

$\mathrm{S} \cdot \mathrm{x}=(\mathrm{xmax}+\mathrm{xmin}) / 2$.;

$\mathrm{S} \cdot \mathrm{y}=(\mathrm{ymax}+\mathrm{ymin}) / 2$.;

// Eulerian Initialization (source point)

XFCT_INIT_EUL_SRCP phiO(S);

ISH* X_eul= new ISH(discrE,phi0);

// Lagrangian Initialization (source point)

XFCT_INIT_LAG_SRCP xsiO(S) ;

ISH* X_lag= new ISH(discrL, Xsi0);

//**** time solver define TSTEP

// euler for Eulerian

TSLV_EUL tseul (eul, discrE);

// RK4 for Lagrangian

TSLV_RK4 tslag(lag,discrL);

// Initial / Final time / constant time step

$R t(0), t 1(3.4), d t(1 e-3)$;

// counter to write solutions at every nexec times step int $\mathrm{cp}(0), \operatorname{nexec}(10)$;

of stream solLag("SOL_lag.dat");

of stream solEul("SOL_eul.dat");

while $(t<t 1)$

\{

cout $<<$ t=" $<$ t $<$ "\n";

$\mathrm{cp}++$;

// Evolution of Lagrangian Sol over one time step tslag.Tstep(t,dt,X_lag);

// Evolution of Eulerian Sol over one time step 
PLOT

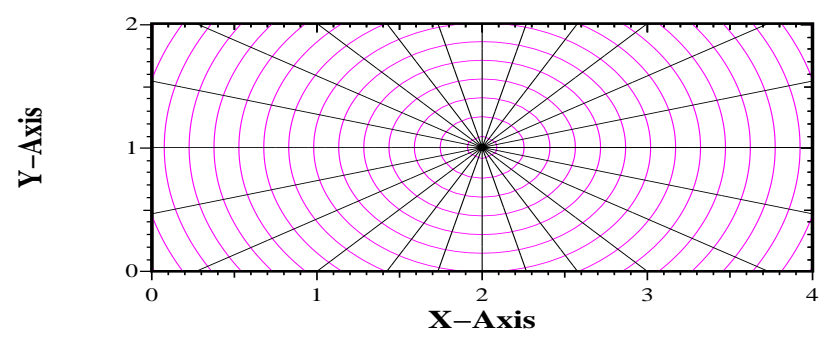

Figure 1. Rays and level curves of the phase.

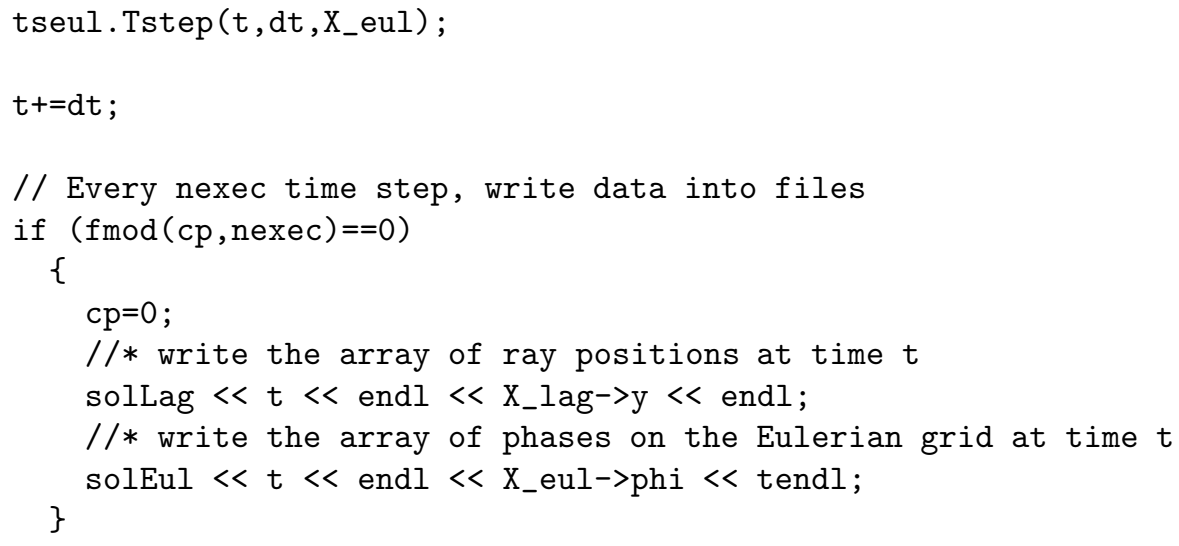

\subsection{Results}

The parameters are $x 1_{\min }=0, x 1_{\max }=4, x 2_{\min }=0, x 2_{\max }=2$. The source point $\mathrm{S}$ is located at the center of the domain. In the Eulerian case, we took $n x_{1}^{\mathrm{e}}=n x_{2}^{\mathrm{e}}=51$, ord $=1$, and for the Lagrangian problem $n x_{1}^{l}=n x_{2}^{1}=5$ which gives 25 rays. We evolve the two problems simultaneously until time $t_{\max }=3.4$ with a constant time step 0.01. The compile time takes about $20 \mathrm{~s}$, and the run time $1.5 \mathrm{~s}$, on a pentium $750 \mathrm{mHz}$.

Figure 1 shows on the same plot the projection of the "space-time rays" on the plane and the zero level curves of $t-\phi(t, x)$ of the Eulerian phase. As expected (the Lagrangian problem can be solved analytically, the phase is single-valued and the Eulerian solution follows automatically) the rays are straight lines and the level curves behave as regular expanding circular wavefronts.

Here we have used plotmtv as a graphical software (see [6] for more on graphic facilities in GO++).

\section{A SECOND EXAMPLE: A WAVEGUIDE PROBLEM}

We review the different steps needed to solve a similar problem set with a different and more general Hamiltonian function and a different numerical method. 


\subsection{Creating a new Hamiltonian class}

Our new Hamiltonian function is the classical geometric optic Hamiltonian with variable index of refraction $n$ :

$$
H(s, y, p)=\frac{1}{2}\left(|p|^{2}-n^{2}(y)\right)
$$

We take

$$
n(x)= \begin{cases}1 & |x|>0.5 \\ 1+\cos ^{2}(\pi|x|) & |x| \leq 0.5\end{cases}
$$

a function of configuration space which can be implemented in a child class of the $X F C T$ class called $X F C T \_I N D E X \_W G$ :

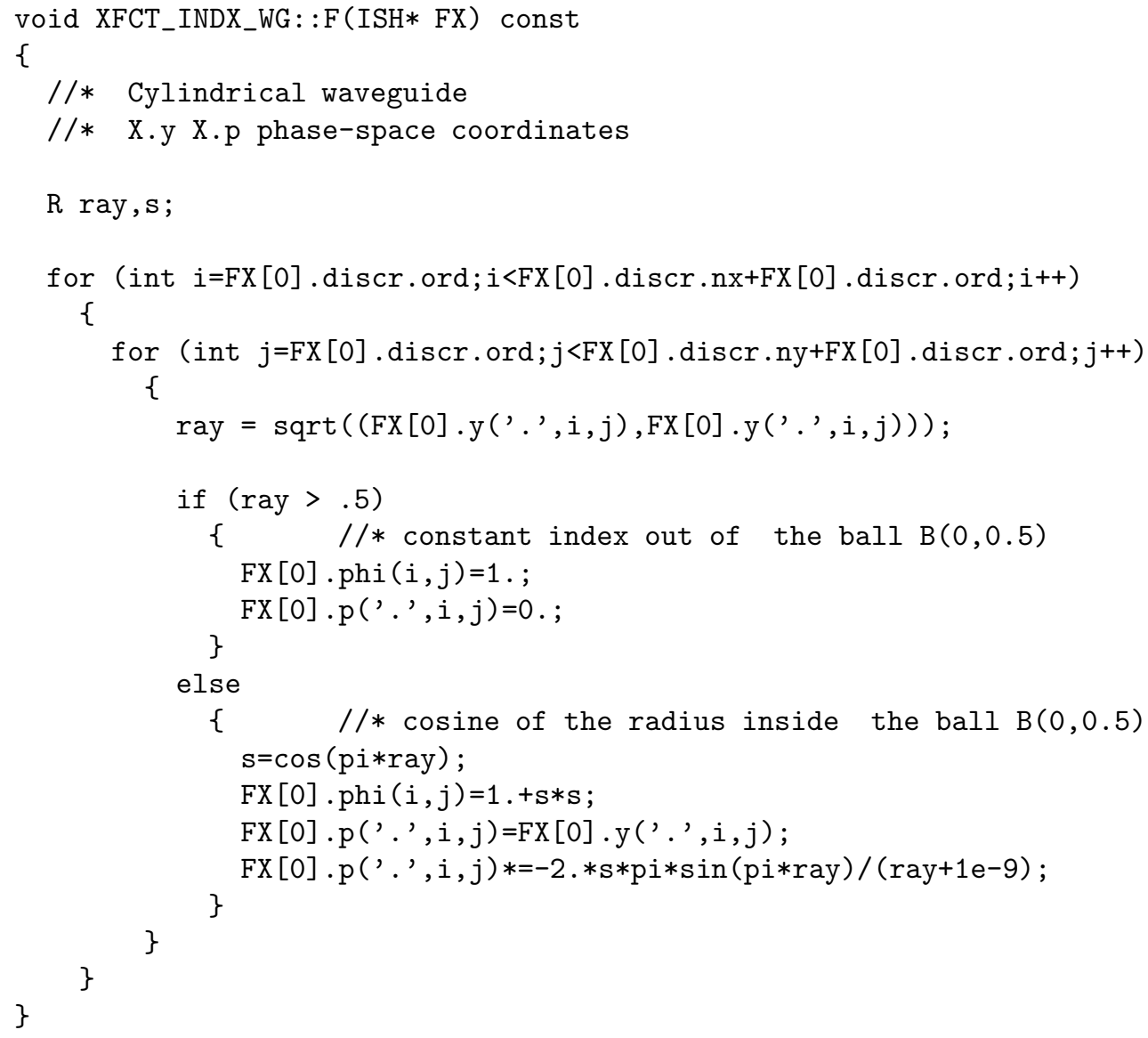

Then we create a child class of $H A M I_{-} C O N T$ which includes the specification of an index of refraction in the form of a XFCT class. This new Hamiltonian class is called HAMI_CONT_XFCT. We specialize further the Hamiltonian class to take into account the particular geometrical optics form (7) in the class $H A M I \_C O N T$ I XFCT_GO.

class HAMI_CONT_XFCT: public HAMI_CONT

\{

public:

//* Specify a function XFF 


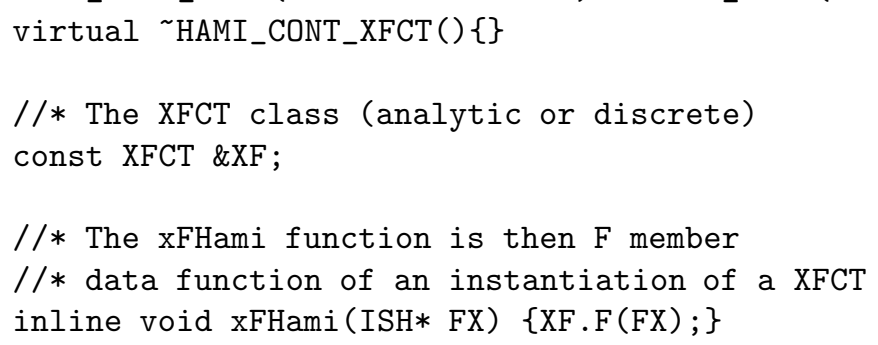

The last step is to modify the declaration lines for the Hamiltonian to make these changes operational, (i.e. modify the declaration in the model section of the main program)

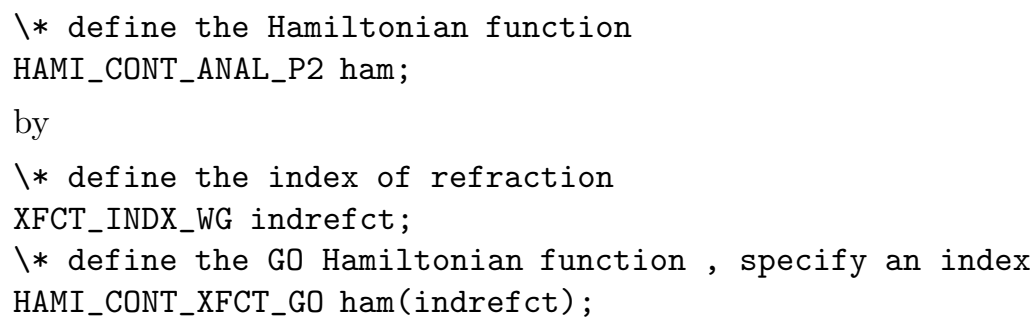




\subsection{Implementing a new numerical Hamiltonian}

The same mechanism is used to modify the numerical Hamiltonian. A Godunov method specialized for convex Hamiltonian depending on $p^{2}$ like for HAMI_CONT_XFCT_GO can simply be written as:

$$
H_{G O D}^{\text {num }}(s, x, a, b, c, d):=H(s, x, \max (\max (a, 0),-\min (b, 0)), \max (\max (c, 0),-\min (d, 0)))
$$

where $(a, b, c, d)$ are defined in Section 7.1. We then create a child class HAMI_NUM_GOD (source below) and modify accordingly the class declaration:

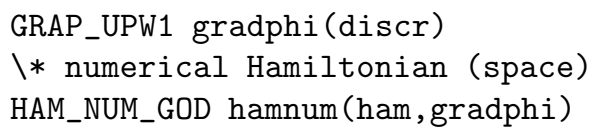

Note that ham describes our new Hamiltonian function and gradphi the upwind formula, the $\mathrm{C}++$ code in this case is:

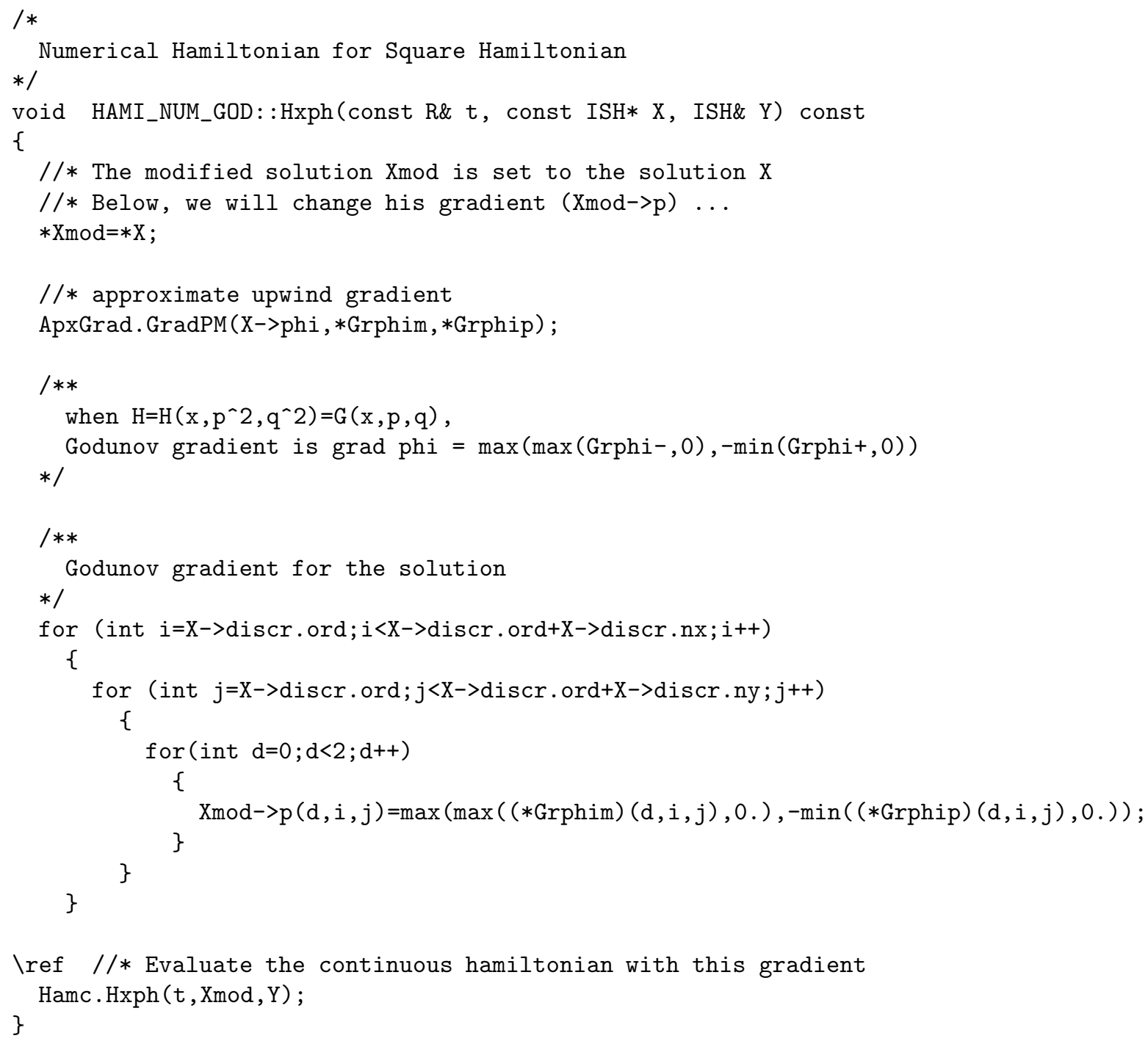




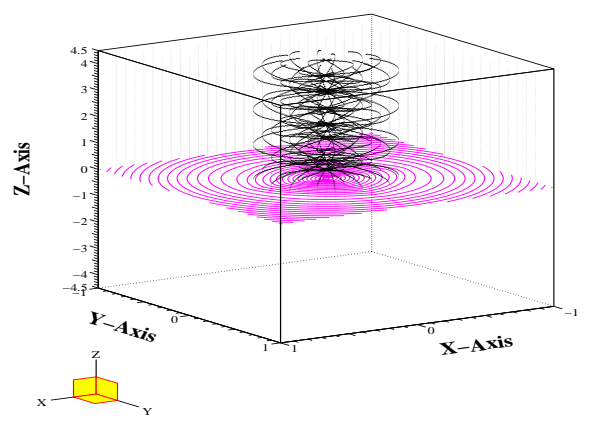

PLOT

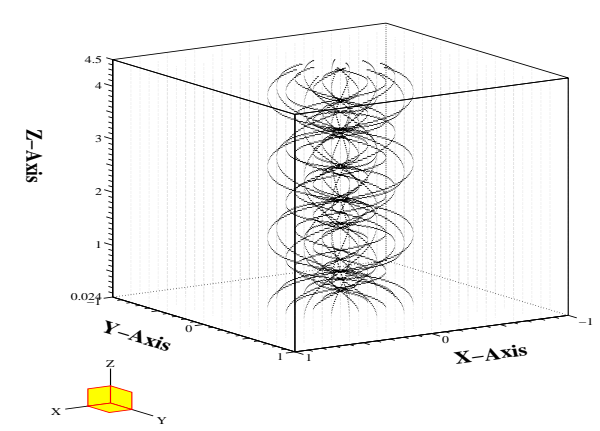

FIGURE 2. Space time perspective of the rays.

\subsection{Changing the initialization}

In Section 4.4, we already created initialization functions (for a source point simulation). One can modify the initialization function by creating his own XFCT child class. For the wave guide simulation the default initialization $\phi^{0}=0$ (straight rays) is used.

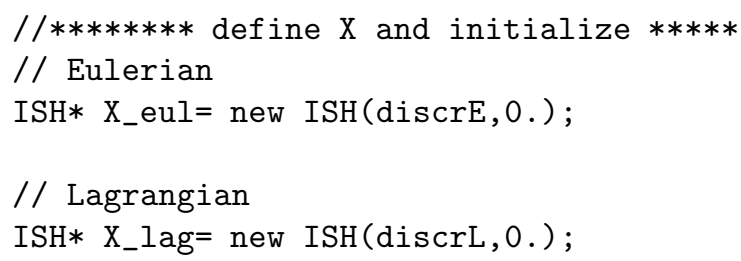

\subsection{Changing the boundary conditions}

As we use a Godunov solver for our numerical Hamiltonian we must modify the boundary conditions accordingly.

// Conditions Limites cas Eulerien (Godunov solver)

BCON_EUL_OUTG_GODUNOV bcond;

\subsection{Results}

The index of refraction has a maximum at $x=0$ and bends the rays towards the $x=0$ line. Shooting straight rays $\left(\phi^{0}=0\right)$ in the interior region $|x|<0.5$ will produce a wave guide effect. In this simulation, $x_{1, \min }=-1, x_{1, \max }=1, x_{2, \min }=-1, x_{2, \max }=1$. In the Eulerian case, we took $n x_{1}^{\mathrm{e}}=n x_{2}^{\mathrm{e}}=101$, ord $=1$, and for the Lagrangian case $11 \times 11$ rays. We evolve the two problems simultaneously until the time $t_{\max }=4.5$ with a constant time step 0.0075 .

In Figure 2 we see the waveguide effect on the rays while outside the zone of inhomogeneity of the index rays propagate in straight lines.

Figure 3 shows two time slices of the phase computed with the Hamilton Jacobi equation. On can observe the radial spatial gradient of the phase corresponding to the index of refraction. The Eulerian solution picks up the phase of only the fastest rays according to formula (4). 

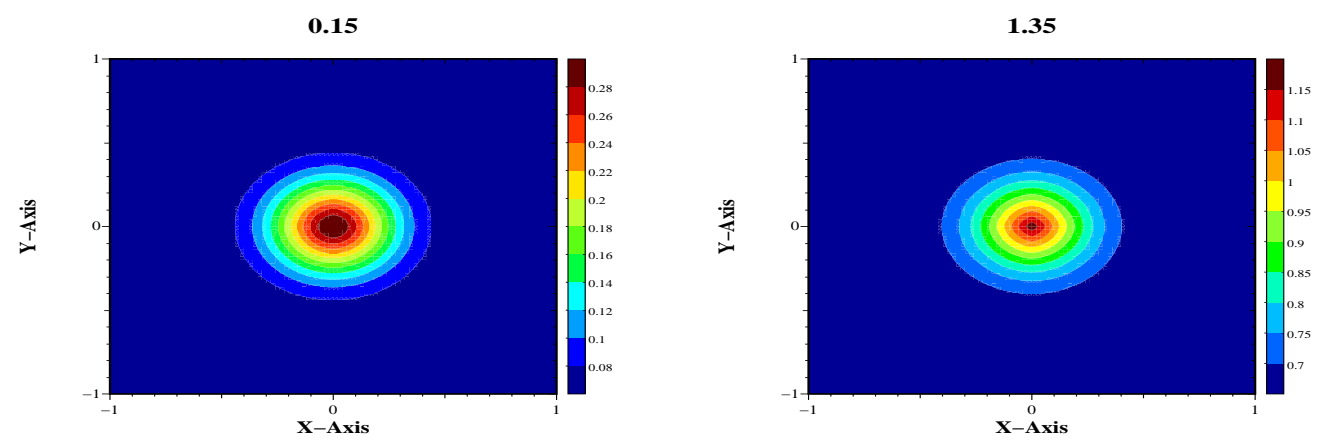

Figure 3. Greyscale map of times slices of the phase.

\section{A generalization to Semi-AnAlytically Defined Hamiltonian}

In many applications the Hamiltonian function reflects the properties of a medium which are not necessarily known analytically. For example the sound speed $c$ in the ground may be specified on a grid like in the Marmousi test case (see http://www-rocq.inria.fr/ ${ }^{\sim}$ benamou/traveltimes.html). In this case, the geometric optics Hamiltonian is $H(s, y, p)=c(y)|p|$ with the sound speed $c$ given as collection of samples at grid points $\left(x_{1, i}^{\mathrm{int}}, x_{2, j}^{\mathrm{int}}\right), i=1, . ., n_{x 1}^{\mathrm{int}}, j=1, . ., n_{x 2}^{\mathrm{int}}$. An interpolation method is therefore needed at least for the Lagrangian resolution and also in the Eulerian case when the Eulerian grid does not match with the location of the samples. This functionality can be integrated into $\mathrm{GO}++$ by the addition of a family of a new class.

\subsection{Interpolation methods: the $X F C T \_I N T R$ and the $I N T R$ classes}

We recall that the $X F C T$ has a child $X F C T \_I N T R$ which stands for functions specified only at a discrete set of points and needs and interpolation method. The $F$ member function of $X F C T$ is then replaced by a member function of INTR called Interp which does the interpolation job. The INTR virtual class has therefore a virtual member function $I n t e r p$ which takes an $I S H$ variable say $I X$ that contains $I X . y=X$ the set of points, $I X . p=\nabla F(X)$ the gradient of the function at these points and $F X . p h i=F(X)$ the function itself.

To build such an object, we must read sample data. We treated the case of data specified on a Cartesian discretization of a square domain in a specialized class called INTR_CART. A text data file (generically called Intrfile.dat) containing $\left.\left(x_{1, \min }^{\mathrm{int}}, x_{1, \max }^{\mathrm{int}}, x_{2, \min }^{\mathrm{int}}, x_{2, \max }^{\mathrm{int}}\right), n_{x 1}^{\mathrm{int}} n_{x 2}^{\mathrm{int}}\right)$ the coordinates and discretization of the sample points followed by the value of the function at these points must be specified. The several methods can be used such as B-splines (INTR_CART_BSPLINE) or Catmull-Romm splines (INTR_CART_CRSPLINE).

In the Marmousi test case the top lines of the Intrfile.dat (called "marmousi.dat") file looks like:

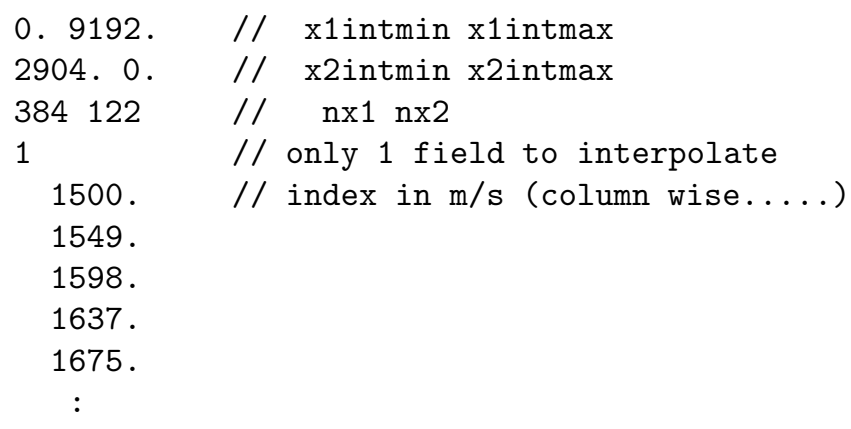




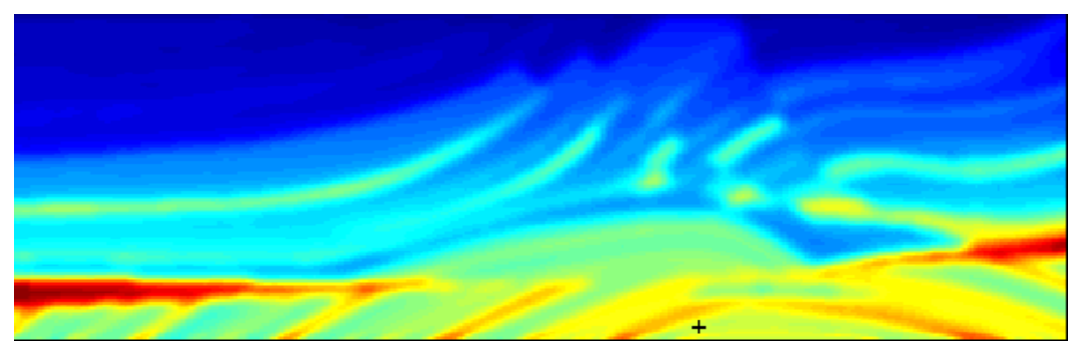

PLOT

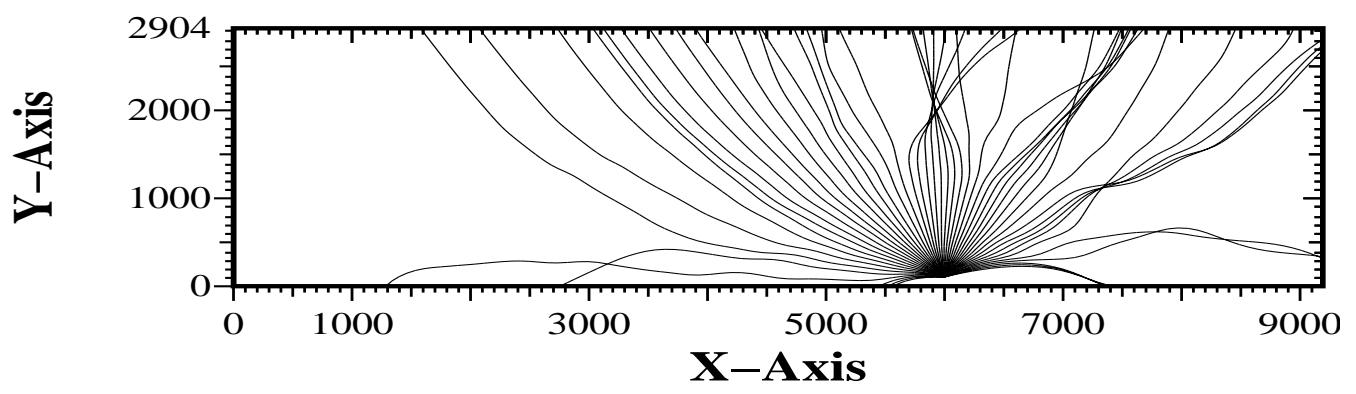

Figure 4. The speed $c$, the point source and the rays.

where the data are respectively: $x_{1, \text { min }}^{\text {int }}, x_{1, \text { max }}^{\text {int }}$, and $x_{2, \text { min }}^{\text {int }}, x_{2, \text { max }}^{\text {int }}$, and $n_{x 1}^{\text {int }} n_{x 2}^{\text {int }}$, and the number of data fields, and finally the values (regularly spaced) of the sound speed.

Then if we want to use B-spline, the syntax are defined by the following specifications:

$\backslash *$ define the interpolation method and data file

INTR_CART_BSPLINE interp("marmsmooth.dat");

$\backslash *$ Define the index

XFCT_INTR marmind(interp);

$\backslash *$ define the Hamiltonian

HAMI_XFCT_CONT_MI ham(marmind) ;

\subsection{Result}

We solve the problem on the domain defined by $x 1_{\min }=0, x 1_{\max }=9192, x 2_{\min }=0, x 2_{\max }=2904$ with a source point $\mathrm{S}$ located at $(6000,104)$. In the Eulerian case, we use the same initial condition as in Section 9 and apply outgoing boundary conditions. The discretization uses $n x_{1}^{\mathrm{e}}=384, n x_{2}^{\mathrm{e}}=122$, and a 2 nd order TVD [15] $($ ord $=2)$ method for the approximation of the gradient.

In the Lagrangian case, we shoot $7 \times 7$ rays, the initial condition is the same as in the isotropic case except that $X . p$ is divided by the sound speed at the source point $\mathrm{S}$. We evolve the two problems simultaneously until the time $t_{\max }=2.6$ with a constant time step 0.0043 .

Figure 4 shows the heterogeneous speed function characterizing the underground, the location of the source point and the associated rays which are bent according to the variation of the index $\frac{1}{c}$. A grayscale plot of two time slices of the Eulerian phase are given in Figure 5. It provides in particular the phase function in shadow regions. 


\subsection{8}

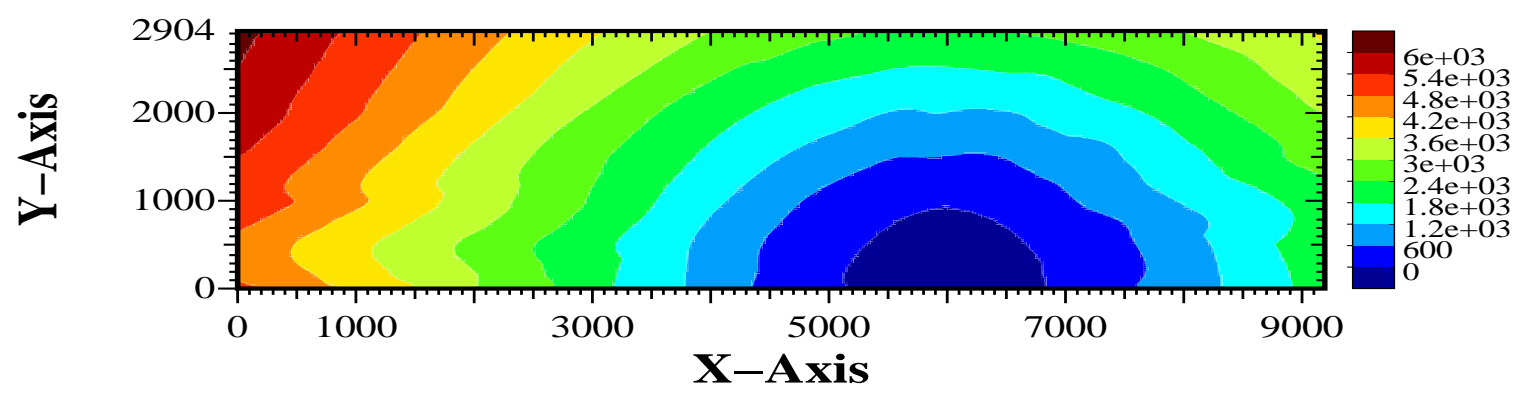

\subsection{6}

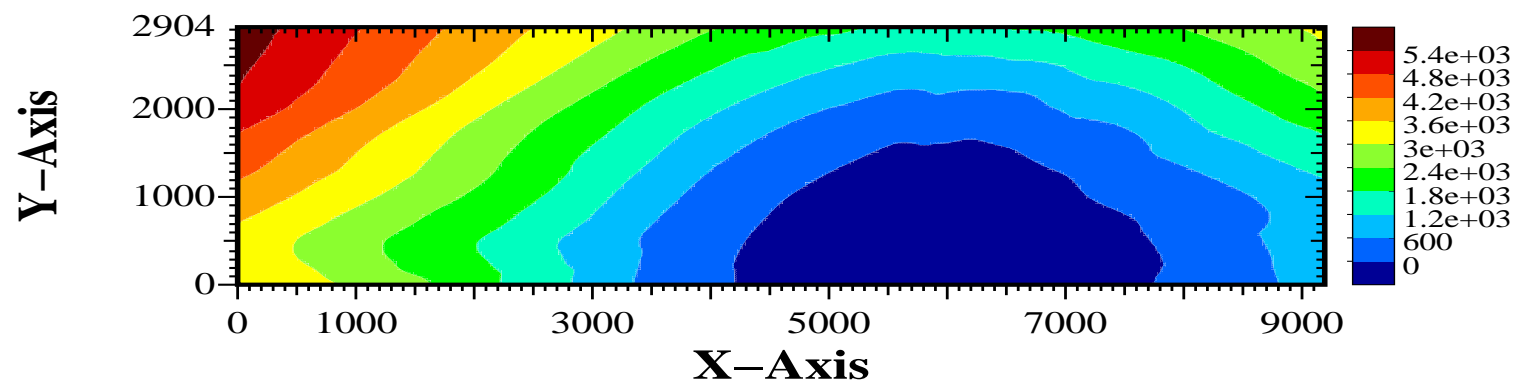

FiguRE 5. Grey scale map of the phase at two different times.

Acknowledgements. Many thanks to S. Delpino and W.W. Symes for their advice on the C++ language.

\section{REFERENCES}

[1] R. Abgrall and J.-D. Benamou, Big ray tracing and eikonal solver on unstructured grids: Application to the computation of a multi-valued travel-time field in the marmousi model. Geophysics 64 (1999) 230-239.

[2] V.I. Arnol'd, Mathematical methods of Classical Mechanics. Springer-Verlag (1978).

[3] G. Barles, Solutions de viscosité des équations de Hamilton-Jacobi. Springer-Verlag (1994).

[4] J.-D. Benamou, Big ray tracing: Multi-valued travel time field computation using viscosity solutions of the eikonal equation. J. Comput. Phys. 128 (1996) 463-474.

[5] J.-D. Benamou, Direct solution of multi-valued phase-space solutions for Hamilton-Jacobi equations. Comm. Pure Appl. Math. $\mathbf{5 2}(1999)$.

[6] J.-D. Benamou and P. Hoch, GO++: A modular Lagrangian/Eulerian software for Hamilton-Jacobi equations of Geometric Optics type. INRIA Tech. Report RR.

[7] Y. Brenier and L. Corrias, A kinetic formulation for multi-branch entropy solutions of scalar conservation laws. Ann. Inst. H. Poincaré Anal. Non Linéaire 15 (1998) 169-190.

[8] M.G. Crandall and P.L. Lions, Viscosity solutions of Hamilton-Jacobi equations. Trans. Amer. Math. Soc. 277 (1983) 1-42.

[9] J.J. Duistermaat, Oscillatory integrals, Lagrange immersions and unfolding of singularities. Comm. Pure Appl. Math. 27 (1974) 207-281.

[10] B. Engquist, E. Fatemi and S. Osher, Numerical resolution of the high frequency asymptotic expansion of the scalar wave equation. J. Comput. Phys. 120 (1995) 145-155.

[11] B. Engquist and O. Runborg, Multi-phase computation in geometrical optics. Tech report, Nada KTH (1995). 
[12] S. Izumiya, The theory of Legendrian unfoldings and first order differential equations. Proc. Roy. Soc. Edinburgh Sect. A 123 (1993) 517-532.

[13] G. Lambare, P. Lucio and A. Hanyga, Two dimensional multi-valued traveltime and amplitude maps by uniform sampling of a ray field. Geophys. J. Int $\mathbf{1 2 5}$ (1996) 584-598.

[14] B. Merryman S. Ruuth and S.J. Osher, A fixed grid method for capturing the motion of self-intersecting interfaces and related PDEs. Preprint (1999).

[15] S.J. Osher and C.W. Shu, High-order essentially nonoscillatory schemes for Hamilton-Jacobi equations. SIAM J. Numer. Anal. 83 (1989) 32-78.

[16] J. Steinhoff, M. Fang and L. Wang, A new eulerian method for the computation of propagating short acoustic and electromagnetic pulses. J. Comput. Phys. 157 (2000) 683-706.

[17] W. Symes, A slowness matching algorithm for multiple traveltimes. TRIP report (1996).

[18] V. Vinje, E. Iversen and H. Gjoystdal, Traveltime and amplitude estimation using wavefront construction. Geophysics $\mathbf{5 8}$ (1993) 1157-1166.

[19] L.C. Young, Lecture on the Calculus of Variation and Optimal Control Theory. Saunders, Philadelphia (1969).

To access this journal online:

www.edpsciences.org 